\title{
Heideggers Denken. Anmerkungen zur Rezeptionsgeschichte der Philosophie ${ }^{1}$
}

\author{
Heidegger's thinking. Remarks on the history of the \\ reception of philosophy
}

Peter Trawny ${ }^{2}$

Bergische Universität Wuppertal, Deutschland

Recepción: 01 de noviembre del 2020

Evaluación: 12 de noviembre del 2020

Aceptación: 20 de noviembre del 2020

${ }^{1}$ Este artículo fue escrito en el marco del trabajo investigativo realizado en el Martin Heidegger-Institut (Martin Heidegger-Forschungsstelle) de la Bergische Universität Wuppertal.

2 Estudió Filosofía, música e historia del arte en la Ruhr-Universität Bochum y en la Albert-LudwigsUniversität Freiburg. Dr. por la Bergische Universität Wuppertal. Posdoctorado y habilitación en la misma Universidad.

Correo electrónico: petertrawny@aol.com

Trawny, P. (2020). Heideggers Denken. Anmerkungen zur Rezeptionsgeschichte der 


\section{Resumen}

El presente artículo parte de la afirmación de que la historia de la filosofía se ha objetualizado en escritos, obras y discursos, que constituyen la base de su recepción. Se indaga en segundo lugar si esta recepción permite captar lo más propio de lo que es la filosofía y no solamente la comprensión de la misma desde la perspectiva europea. Con base en este punto de partida se reflexiona en torno al carácter propio de la filosofía en Martin Heidegger, resaltando la influencia de ésta más allá de su recepción académico-institucional (caso de los Cuadernos negros, por ejemplo). Los puntos fundamentales que se enuncian al respecto son: a) no se puede decir que Heidegger tenga una filosofía, pues él mismo no admite etiqueta alguna, b) si la actividad filosófica se convierte en una profesión -se institucionaliza-, cuya técnica consiste en poner simplemente en relación la lectura de textos, se pierde allí lo que realmente debe acontecer: el pensar mismo, c) es por ello que con el pensar mismo no se trata de resultados alrededor del conocimiento, sino de caminos y encaminamientos (Be-Wegung), d) tal pensar no produce conceptos estables, sino términos que en el movimiento del pensar se transforman y conducen a neologismos, e) de esta manera el asunto del pensar es un NichtSache o un Un-Sache, algo no señalable, f) en la pregunta por el sentido del ser se despliega el espacio y el tiempo en el que se puede encaminar el pensar.

Palabras clave: Heidegger, objetualización de la filosofía, pensar, encaminar (Be-Wegung), Nicht-Sache.

\section{Abstract}

The present paper starts on the affirmation that the history of philosophy is been objectified in writings, works and speeches, which constitute the basis of its reception. The second point inquires if this reception allows us to grasp the most characteristic of what philosophy is, and no only the understanding of it from the European perspective. Based on this starting point we reflect around the character of philosophy in Martin Heidegger, highlighting its influence beyond academic-institutional reception (the case of the black notebooks, for example). The fundamental points that are enunciated in this matter are: a) it cannot be said that Heidegger has a philosophy, since he himself does not admit any label, b) if the philosophical activity becomes a profession -it is institutionalized-, whose technique consists of simply putting in relation the reading of texts, what really must happen is lost there: c) that is why thinking itself is not about results around knowledge, but about paths 
and routes (Be-Wegung), d) such thinking does not produce stable concepts, but words that in the movement of thinking are transformed and lead to neologisms, e) in this way the matter of thinking is a Nicht-Sache or an UnSache, something not to be pointed out, f) in the question of the sense of being unfolds the space and time in which thinking can be directed.

Keywords: Heidegger, objectification of philosophy, thinking, Be-Wegung, Nicht-Sache. 
Die Geschichte der Philosophie ist - auf den ersten und zweiten Blick geseheneine der Texte und Rezeptionen. Philosophen und seit ungefähr einem Jahrhundert Philosophinnen sind auf das Medium der Schrift verwiesen, um ihre Gedanken vor dem Vergessen zu sichern und zu archivieren. Platons Schriftkritik bzw. Sokrates' Schriftverweigerung haben zwar ein kritisches Bewusstsein in Bezug auf die Schriftlichkeit des Denkens ermöglicht, doch die institutionalisierte Philosophie kann ohne Texte und ihre philologische Bearbeitung -ohne das Archiv ihrer Geschichte- nicht existieren.

Das ist nicht unwichtig: Philosophie, welche auch immer, anerkennt die Notwendigkeit, sich in Texten zu manifestieren. Nur so kann sie dauerhaft und unverändert einer beliebigen Leserschaft zugänglich sein, nur so via Übersetzungen jeweils in anderen Ländern, auf anderen Kontinenten, in anderen Sprachen rezipiert werden. Nicht zu Unrecht spricht Marx an exponiertester Stelle von der Entstehung der „Weltliteratur“ (Marx und Engels, 2010) $)^{3}$. So wäre zu fragen und wurde gewiss schon gefragt, ob nicht die Kolonisierung der Welt von Europa her auch eine Kolonisierung durch Schrift gewesen ist (Derrida, 1983) - und welche Rolle die philosophischen Texte bei dieser Kolonisierung gespielt haben. Überhaupt wäre zu fragen, welche Bedeutung der Text, das Buch, für die Verbreitung einer bestimmten Auffassung von Geist und Bildung in der Welt hatte oder noch hat. Inzwischen ist die Diskussion zur Frage nach den Vorzügen oder Nachteilen der Digitalisierung übergegangen. Zweifellos ist das World Wide Web ein anderes Medium als die Schrift und das Buch. Die Veränderungen in der Rezeption von Philosophie und Literatur sind noch diffus, aber spürbar. Doch das ist anderswo zu reflektieren.

Die Festlegung der Geschichte der Philosophie als eine von Text und Schrift bedeutet in sich die Favorisierung des "Werks“ oder auch des „Diskurses“ als ihre zentralen Erscheinungsformen. Philosophie vergegenständlicht sich demnach, muss sich vergegenständlichen, um überhaupt das Objekt einer Rezeption werden zu können. Diese Tendenz der Philosophie zu ihrer eigenen Verobjektivierung ist ein Problem. Es erinnert an die Frage, was Philosophie sei.

3 „Die Bourgeoisie hat durch die Exploitation des Weltmarkts die Produktion und Konsumtion aller Länder kosmopolitisch gestaltet. (...) Die nationale Einseitigkeit und Beschränktheit wird mehr und mehr unmöglich, und aus den vielen nationalen und lokalen Literaturen bildet sich eine Weltliteratur". 
Mich interessiert, welche Rezeptionshaltung und -gewohnheit sich aus diesen Verobjektivierungen für die Philosophie ergab und ergibt, wie sich also schon seit Jahrhunderten ein Zugang zur Philosophie sedimentiert, der die Schrift(en), den Text, das „Werk, den „Diskurs“, in ihre Mitte stellen. Darüber hinaus möchte ich fragen -naheliegender Weise-, ob das Innerste der Philosophie selbst, das, was sie nicht nur sein will, sondern eigentlich auch sein muss, um sie selbst zu sein, in dieser Rezeptionssedimentierung erfasst oder auch nur berührt wird. Ich möchte dabei keineswegs allein auf das europäische Verständnis einer zum Wissen (epistéme) tendierenen Philosophie hinaus. Mir ist bewusst, dass die Redeweise von einem „Innersten der Philosophie“ -gleichsam von ihrer Seele- nicht unproblematisch ist. Doch ich möchte an ihr festhalten.

Um diesen Fragen näher zu kommen, möchte ich mich auf einen Vorgang beziehen, der in der Geschichte der Philosophie des 20. und 21. Jahrhunderts gewiss nicht allzu häufig $\mathrm{zu}$ finden ist. Es geht mir um die vor allem institutionelle Rezeption der Philosophie Martin Heideggers; allerdings eine Rezeption, die auch über die Grenzen der institutionell-akademischen Wahrnehmung hinaus eine Wirkung entfaltet hat. Meine These ist die, dass die Veröffentlichung der Schwarzen Hefte und auch der vielen Anmerkungen zu Sein und Zeit (vor allem im Band 82 der Gesamtausgabe) (Heidegger, 2017) auf einzigartige Art und Weise die Rezeption dieser Philosophie verändert haben. Dieser Vorgang oder diese Veränderung wirft wiederum ein Licht auf das angesprochene Problem der Art und Weise, wie Philosophie überhaupt rezipiert wird und werden kann.

Als 2013/14 die ersten später von Heidegger selbst so genannten Schwarzen Hefte erschienen, drehte sich die hektische Diskussion vor allem um die in ihnen befindlichen antisemitischen Äußerungen. Sie spaltete die Diskutierenden mehr oder weniger in drei Lager: Da waren die orthodoxen Heidegger-Apologeten, die, wie Friedrich-Wilhelm von Herrmann ${ }^{4}$ (von Herrmann und Alfieri, 2017), überhaupt den Status der Schwarzen Hefte als ernstzunehmenden Werkteil im Heideggerschen Denken bezweifelten und die sich auf Juden und Judentum beziehenden Passagen nachgerade für berechtigte „Juden-Kritik“5 (Vietta, 2015) hielten; dann gab es die

\footnotetext{
${ }^{4}$ Bereits der Titel verkündet ein Dogma, das nichts mit Heideggers Denken zu tun hat.

5 Um einen Überblick über die verschiedenen Positionen in der mehr oder weniger aktuellen Diskussion zu gewinnen, sei auf die Aufsatzsammlung Heidegger und der Antisemitismus. Positionen im Widerstreit. Mit Briefen von Martin und Fritz Heidegger (2016) hingewiesen.
} 
orthodoxen Heidegger-Kritiker, die, wie Emmanuel Faye (2005) schlechthin Heideggers gesamtes Denken im Antisemitismus und Nationalsozialismus kulminieren ließen; ein dritter Zugang versuchte, die antisemitischen (oder nicht-antisemitischen) ${ }^{6}$ (Chighel, 2020) Gedanken ernstzunehmen, ohne sie zum Kriterium einer Generalabrechnung zu erheben. Er wurde von den beiden anderen Auslegungen jeweils der gegnerischen Seite zugerechnet. In beiden dogmatischen Positionen sind ideologische Momente unübersehbar. Nicht unwahrscheinlich, dass sie zu einer größeren gesellschaftlichen Spaltung gehören.

Der Streit um Heideggers Positionierung zum Judentum selbst hatte einen Einfluss auf die akademische Rezeption dieses Denkens. In Zeiten von „Political Correctness“ und „Cancel Culture“ ist es unwahrscheinlich, dass die zunächst öffentliche Abkanzelung eines Philosophen ohne Effekt auf seine akademische Rezeption bleibt. Es ist daher in Deutschland schwieriger geworden, Heideggers Denken jenseits moralisch-politischer Vorurteile oder Urteile zu studieren. Das mag in anderen Ländern, anderen UniversitätsKulturen anders sein. In China haben Antisemitismus-Diskussionen eine andere Bedeutung als in Europa.

Ich bin jedoch der Ansicht, dass die Heidegger-Rezeption weniger durch die moralisch-politischen Stellungnahmen des Philosophen, sondern mehr noch durch den in den Schwarzen Heften neu erprobten Stil des Denkens, seine neue, bisher unbekannte Äußerungsform, erschüttert wurde. Denn mit der Veröffentlichung der neun Bände Schwarze Hefte in Heideggers Gesamtausgabe (sowie seinen Selbstauslegungen von Sein und Zeit) wird deutlich, dass die Positionierung des Heideggerschen Denkens um ein oder zwei Hauptwerk(e), um Sein und Zeit und die Beiträge zur Philosophie, dem Charakter dieses Denkens nicht entspricht.

Dabei hatte Heidegger schon früh begonnen, seine Rezeption zu kritisieren. In der Vorlesung Der Anfang der abendländischen Philosophie vom Sommer 1932 teilt er mit:

Ich habe keine Etikette für meine Philosophie, und zwar deshalb nicht, weil ich keine eigene Philosophie habe; ja überhaupt keine Philosophie, sondern es handelt sich bei meinen Bemühungen nur darum, den Weg dahin zu erobern

\footnotetext{
6 Wer meine Auslegung von Heideggers „,seinsgeschichtlichem Antisemitismus“ verstehen und kritisieren will, muss dieses ausgezeichnete Buch, das ich aus dem Englischen übersetzte, lesen.
} 
und zu bereiten, daß Künftige vielleicht wieder mit dem rechten Anfang der Philosophie anfangen können (Heidegger, 2012)

Heidegger habe nicht nur „keine eigene“, sondern „überhaupt keine Philosophie“. Das sagt er ein halbes Jahrzehnt nachdem er sich mit der Veröffentlichung von Sein und Zeit an die Spitze nicht nur der deutschen und europäischen Philosophie katapultiert hatte. Auch in Südamerika und im fernen Asien begann man, Heidegger zu rezipieren.

Dass ein Philosoph „überhaupt keine Philosophie“ vertrete, klingt zunächst rhetorisch. War Heidegger nicht der berühmte Professor der Philosophie an der Freiburger Universität, deren Rektor er im nächsten Jahr werden sollte? Saßen nicht Massen von Studenten und Studentinnen in seinen Vorlesungen und Seminaren, die seinen Worten folgten? Waren seine Vorträge nicht Ereignisse eines nachgerade öffentlichen Interesses? Heidegger gehörte zur Institution der deutschen Universität, zum „Establishment“, und doch sind seine Worte, er vertrete „überhaupt keine Philosophie“ nicht falsch. Sein Hinweis, er arbeite dafür, „daß Künftige wieder mit dem rechten Anfang der Philosophie anfangen können“, dass also „überhaupt“" wieder mit der Philosophie angefangen werden könne, bezieht sich auf jene Differenz im Denken, von der beiläufig am Beginn des Textes die Rede war.

Heidegger wurde nicht nur Anfang der Dreißigerjahre häufig mit Karl Jaspers in einem Atemzug als „Existenzphilosoph“ bezeichnet. Dass Heidegger im Sommer 1932 darauf verweist, „,keine Etikette“ für sein Denken zu haben, kann eine Reaktion auf die Publikation der dreibändigen „Philosophie“ von Jaspers im selben Jahr sein. Inzwischen wird allgemein anerkannt, dass Heideggers und Jaspers' Projekte stark divergieren. Doch Heidegger will mehr als sich einem modischen Diskurs zu entziehen. Er betont, dass die Vergegenständlichung der Philosophie im Text ein Problem darstellt. Mit ihr entsteht ein Objekt, ein „Werk“, das im Prozess der Lektüre angeeignet werden kann. Wenn nun diese Tätigkeit zu einem Beruf wird und die Technik dieses Berufes darin besteht, Lektüren von Texten miteinander zu verbinden, geht verloren, was sich jeweils vor und nach der Sedimentierung des Gedankens im „Werk“ ereignet: das Denken selbst.

Das war es ja, was Platon mit seiner Schriftkritik im Phaidros sagen wollte:

O kunstreicher Theut. Einer weiß, was zu den Künsten gehört, ans Licht zu gebären; ein Anderer zu beurteilen, wieviel Schaden und Vorteil sie denen bringen, die sie gebrauchen werden. So hast du auch jetzt als Vater der 
Buchstaben aus Liebe das Gegenteil dessen gesagt, was sie bewirken. Denn diese Erfindung wird der [sic] Lernenden Seelen vielmehr Vergessenheit einflößen aus Vernachlässigung des Gedächtnisses, weil sie im Vertrauen auf die Schrift sich nur von außen vermittelst fremder Zeichen, nicht aber innerlich sich selbst und unmittelbar erinnern werden (Platon, 2017, 274e-275a).

So berichtet Sokrates eine ägyptische Geschichte von der Begegnung zwischen dem Gott Theut und dem König Thamos, ohne Zweifel ein ehrwürdiger Mythos für den griechischen Philosophen. Die Schrift bringt Vergessenheit (léthe), sie behindert das Erinnern (anámnesis) -und was ist das Denken anderes als Erinnern? Wäre dann nicht die „Seinsvergessenheit“ eine Art von „Denk-Vergessenheit“, ein Missverständnis des Denkens, das so früh schon, am Anfang der Philosophie, zu wirken begann?

Erstaunlich ist, dass in jener Äußerung von Heidegger aus dem Sommer 1932 en passant schon das Wort erscheint, das er später auf vielfältige Art und Weise zur Charakterisierung seines Denkens verwendet hat: es handele „sich bei meinen Bemühungen nur darum, den Weg dahin zu erobern und zu bereiten, daß Künftige vielleicht wieder mit dem rechten Anfang der Philosophie anfangen können" (kursiv von mir). Ungefähr seit Mitte der Fünfzigerjahre beginnt Heidegger, das semantische Feld des „Weges“, der „Bewegung“, auch des „Gehens“ und des „Gangs“ zu bearbeiten. Das Motto der Gesamtausgabe, „Wege - nicht Werke“ (Heidegger, 1978), verweist zurück auf eine lange Beschäftigung und einen ausgiebigen Gebrauch dieser Entgegensetzung.

Was ist die, wie es in „Winke I“ vom Ende der Fünfziger Jahre heißt, „innerste Be-wēgnis des Denkens“? (Heidegger, 2020a, p. 43) Heidegger hat die Bedeutungen des „Weges" bis in die Titel seiner „Werke“ hinein sehr ernst genommen (Holzwege, Wegmarken, Der Feldweg, Unterwegs zur Sprache etc.). Das auch graphisch markierte „Be-wëgen“ (Heidegger, 1985, p. 199) eröffnet Wege des Denkens, die nicht durch Ziele definiert werden. Heidegger ist sich der Besonderheit einer solchen Auffassung von Philosophie bewusst, wenn er schreibt:

Wege -Vielleicht ist die Blindheit für dergleichen wie Wege des Denkens für das Denken, das als Denken unterwegs - und wege-bauend ist -das ärgste Hindernis für ein fruchtbares Gespräch mit meinen Denkversuchen. Man bringt es nicht über sich, sich auf den Weg zu begeben-. Man will Ergebnisse und zwar nach irgendwelchen Kriterien der Sicherung (Heidegger, 2019, p. 190). 
Tatsächlich scheint die Rede von „Wegen“, so einleuchtend ihre Rückführung auf die griechische Bedeutung der Methode ist, nicht unproblematisch $\mathrm{zu}$ sein. Zunächst ist es nicht zu übersehen, dass Heidegger von „Wegen“ und nicht einfach, wie ein in der Forschung bekannter Buchtitel es tut, von dem „Denkweg“ (Pöggeler, 1963) spricht. Das Denken bildet nur insofern eine Kontinuität aus, als es synchron verschiedene „Wege“ bildet und verfolgt. Dieser Aspekt der konkreten philosophischen Arbeit hat sich bei Heidegger darin niedergeschlagen, dass er häufig an verschiedenen Manuskripten gleichzeitig gearbeitet hat, die oftmals stilistisch große Unterschiede aufweisen.

Zudem hebt die Differenzierung von „Weg“ und „Ergebnis“, d.h. Ziel, das Denken in eine eigentümliche Schwebe. Warum denken wir überhaupt, wenn es nicht darum geht, bestimmte Denkprobleme zu lösen? Das ist es nämlich, was Heideggers Denken immer wieder betont. Es gehe nicht um Resultate, um Erkenntnisse, sondern um die „Be-Wegung“ (Heidegger, 2019, p. 214) selbst:

An meinem Weg verkennt man bisher immer wieder -wissentlich oder unwissentlich-zwei wesentliche Be-stimmungen:

1. daß dieses Denken überall und stets sich als vor-läufiges versucht;

2 daß in dieser innegehaltenen Vorläufigkeit die ständig ursprüngliche Selbstkritik verankert ist (p. 55),

schreibt Heidegger in einem der späteren Schwarzen Hefte. Ein „,vor-läufiges“ Denken ist eines, das sich „vor“ bewegt, dass in diesem Sinne vor-denkt. Zugleich ist es stets revidierbares, niemals vollendetes Denken. Das kann dieses Denken sein, weil es in einer „ständig ursprünglichen Selbstkritik verankert" ist. Die Kritik kommt demnach nicht später zum Denken hinzu, sondern bildet schon seinen Anfang. Offenheit, von der Heidegger so häufig spricht, ist kein Ziel des Denkens. Sie ist seine Realisierung, sein Vollzug. Und wenn das Denken von Beginn an Kritik ist, dann ist sie Offenheit. Und was sollte Kritik anderes sein?

„Innerste Be-wēgnis des Denkens“ ist gedankliche Bewegung als intellektuelle Intimität. Dieses Denken befindet sich immer „kritisch“ in Distanz und Differenz zu sich selbst, es nimmt seine sprachliche Äußerungsweise als Feld jener Bewegung. Daraus entspringt übrigens die offenbare Unbegrifflichkeit dieses Denkens. Es schafft keine stabilisierenden „Begriffe“, sondern arbeitet 
sich an „Worten“ ab, die in der Bewegung des Denkens eine Veränderung erfahren. Dieses Arbeiten in und an den Bedeutungen, die sich wiederum als „Worte“ manifestieren, erscheint zuweilen als eine sinnlose Produktion von Neologismen, in der jeglicher Gegenstand des Denkens verloren geht. Doch dieses Denken hat von vornherein keinen Gegenstand.

Heidegger hat die von Husserl formulierte Parole „Zu den Sachen selbst!“ modifiziert, indem er von der „Sache des Denkens“(Heidegger, 2007) spricht. Der Genitiv ist zweideutig. Er besagt, dass es eine vom Denken bearbeitete „Sache“ gibt, wie dass diese „Sache“ das Denken selbst ist. Wie auch immer Heidegger sonst noch die „Sache“ erläutert hat, so scheint die Redewendung doch eine Bedeutung zu konstatieren, die es in Heideggers Denken nicht geben kann.

Wenn man ernstnimmt, was Heidegger über das „Be-wëgen“ des Denkens sagt, dann kann Sein und Zeit, können die „Beiträge zur Philosophie“ kein „Hauptwerk“ sein, sondern eben „Wege“ unter vielen. Dennoch kommt dort, in Sein und Zeit, jene Trope von der „Frage nach dem Sinn von Sein“ (Heidegger, 1977, p. 1) zum ersten Mal zum Vorschein. So sehr diese Frage gleichsam dogmatische Bedeutung hat, da sie z.B. in akademischen Kontexten als „Heideggers Frage“ gelehrt werden kann, so wenig ist jemals deutlich geworden, was sie besagt.

Zwar wurde in Sein und Zeit selbst mit dem Hinweis auf die „Zeitlichkeit“" des „Daseins“ eine Antwort angedeutet. Doch schnell wurde klar, dass diese nur provisorisch war. Gewiss hat der Philosoph versucht, die Frage zu erläutern, indem er den „Sinn“ als die „Wahrheit des Seins“ bestimmte. Doch haben wir die „Frage nach dem Sinn von Sein“ verstanden? Hat er sie verstanden? Geht es in diesem Denken ums ,Verstehen“? War nicht gerade die Dunkelheit der Frage derartig produktiv, dass sich ein Denken Jahrzehnte lang an ihr abarbeiten konnte? Es geht nicht nur darum, zu verstehen, dass das Denken „unverständlich“ ist (Heidegger, 2015, p. 373) ${ }^{7}$, sondern dass es in der „Seinsfrage“ um etwas anderes als die Verständlichkeit geht. So schreibt Heidegger: „Die Seinsfrage in Sein und Zeit sucht als Erfragen der Wahrheit des Seins nicht eine Antwort auf eine Frage, sondern sucht das fraglose eigentliche Antworten als Gehören in die ,Wahrheit‘ als dem Seyn des Seins“" (p. 274).

7 Heideggers Bestreitung der „Verständlichkeit“ es Denkens ist notorisch. 
Das Denken bezeugt seine Zugehörigkeit zu dem, was es denken lässt. Die Zugehörigkeit ist „fraglos“, weil sie das Denken erst ermöglicht. Um diese Ermöglichung geht es im Denken beinahe allein. Sie erweist sich demnach als „Wahrheit" im Sinne jenes zusammengehörenden Wechselspiels von Verbergung und Entbergung. Gerade das Verborgene (die léthe) -was niemals zur Erscheinung kommen kann- gibt Denken frei. Was schon verstanden ist, blockiert das Denken. Was zu verstehen sein wird, wird es blockieren. Nur das Unverstehbare lässt gänzlich ungehindert denken.

Ein weiterer Lehrgehalt dieses Denkens ist die aus der "Seinsfrage“ selbst folgende „ontologische Differenz“. Das „Sein selbst“" sei „nichts Seiendes“. Über Jahrzehnte hinweg wurde diese „Differenz“ zum Angelpunkt des Heideggerschen Denkens erklärt. Die Rezeption von Heideggers Philosophie drehte sich nicht selten einzig um sie (Trawny, 2016, pp. 59-68). Doch auch diese Denkfigur hat Heidegger später kritisiert, wenn auch offen gehalten: „Also ist der Weg durch die Differenz irrig und darum vergeblich. Gleichwohl wurde der Gedanke an die Differenz als solche zum Anlaß, den Denkblick für ganz Anderes -in der ,Seinsfrage‘ dunkel Gesuchtes- zu öffnen und frei zu halten“".

Das „Sein selbst“" als das ,ganz Andere“"zu denken, führt zu einer Philosophie, in der „Verdinglichung“ notwendig ein zentrales Thema wird. Schon sehr früh, noch vor Lukacs' „Geschichte und Klassenbewußtsein“, warnt Heidegger in einer Vorlesung vor der wissenschaftlichen „Verdinglichung“ des ungegenständlichen Lebens (des biographischen, nicht biologischen) in seiner stets selbstbezogenenen Verwirklichung. Wie ist eine notwendig verobjektivierende Wissenschaft vom Leben möglich, wenn dieses niemals Gegenstand sein kann? Die Denkfigur ist bereits aus der „Negativen Theologie“ vertraut. Das „Leben“ oder „Sein selbst" ist -weil jenseits aller Gegenständlichkeit, jenseits des Seienden-namenlos. Jeder Sinn verstellt es, entfremdet es von sich selbst ${ }^{8}$.

8 Heidegger fragt sich, was Wissenschaft als Denk- und Sprachform notwendig voraussetzt. So findet er eine „Leitidee der Wissenschaft überhaupt und jeder Wissenschaft“. 1919/20 nennt er sie: die „Idee der Dinglichkeit“ oder, besser, „Verdinglichung“ (Heidegger, 1993, p. 127). Vgl. auch Martin Heidegger: Zur Bestimmung der Philosophie. 1. Die Idee der Philosophie und das Weltanschauungsproblem. 2. Phänomenologie und transzendentale Wertphilosophie (1999, p. 66). „Verdinglichung“ -es ist ein historisches Rätsel, woher Heidegger im Winter 1919 diesen Begriff hat. Er verwendet ihn auch in „Sein und Zeit“. Man hat einen Einfluss von Lukacs” „Geschichte und Klassenbewußtsein“ angenommen, doch das Buch erscheint erst 1923. Sollte Heidegger 1919 
In seiner Antrittsvorlesung von 1929 „Was ist Metaphysik?“ hat Heidegger der Wissenschaft vorgeworfen, keine Ahnung vom ,nichtenden Nichts“ zu haben (Heidegger, 1976, p. 106) ${ }^{9}$. Das ist natürlich nur allzu richtig. Eine Wissenschaft vom Nichts würde schnell an ihr Ende gekommen sein. In der positivistischen Ausrichtung der modernen Wissenschaft hat eine solche Formulierung geradezu etwas Komisches, sie lädt zu absurden Ausschweifungen ein (Drittmittelforschung zum oder auch für Nichts...). Für die Philosophie gilt das Gegenteil. Sie thematisiert das Nichts im eminenten Sinne.

Heideggers Denken bewegt sich im Ungegenständlichen und findet dafür immer wieder neue Sprech- und Schreibfiguren. Die „Sache des Denkens“ ist demnach ohne Zweifel eine Un- oder Nicht-Sache, nichts, worauf das Denken zeigen oder aufbauen könnte. Es liegt auf der Hand, zwischen der „,innersten Be-wēgnis des Denkens“ und dieser Ungegenständlichkeit seiner „Sache“ einen Zusammenhang zu sehen. Das Denken kommt deshalb nicht zu Resultaten, weil jedes Resultat zum Gegenstand gerinnt. Nicht auf Resultate gerichtet, sind die „Wege“ dieses Denkens ziellos.

Man kann fragen, ob dieses gegenstandslose Denken sich darum dreht, die „Bedingungen der Möglichkeit“ des „Seienden“ zu erfassen. Das scheint letztlicheinemetaphysischeDenkformzusein, dieHeideggergeradeunterlaufen wollte. Eine solche ,transzendentale“ Begründung des Philosophierens hat er in Sein und Zeit angetroffen, um sie zu kritisieren. Ein Denken, das auf „Bedingungen der Möglichkeit“ aus ist, bleibt gegenstandsbezogen. Heideggers Denken destruriert solche Begründungsfiguren.

Vielleicht nicht überall. Doch ich würde dafür votieren, dass dort, wo Heidegger z.B. zu massiven kulturkritischen Bemerkungen kommt, er hinter seinen eigenen Anspruch, das ungegenständliche „Sein selbst“" (in welcher Bedeutung auch immer, z.B. in der des „Ereignisses“) zu denken, zurückfällt.

schon eine Marx-Lektüre hinter sich gehabt haben? Wenig wahrscheinlich. Unbezweifelbar aber ist, dass er den Begriff recht oft benutzt und wichtig findet. Übrigens ist Heidegger natürlich nicht der Einzige, der auf den primär ungegenständlichen Charakter der Philosophie hingewiesen hat. All jene Philosophen wie z.B. Pierre Hadot oder heute anscheinend Giorgio Agamben, die Philosophie als „Lebensform“ charakterisieren, sind wie Heidegger Kritiker der Vergegenständlichung des Philosophierens. Dennoch setzt Heidegger seine Kritik anders an -wenn auch das „Leben“ bei ihm von Anfang an eine wichtige Rolle gespielt hat. Vgl. Trawny (2019).

9 „Die Wissenschaft will vom Nichts nichts wissen“. Bei solchen Sätzen beginne ich daran zu zweifeln, dass Heidegger kein Ironiker gewesen sein soll. 
Denn wie sich das „Sein selbst“ (oder das „Ereignis“) in spezifischen Kulturgestalten manifestiert, ist eine Frage, die auf jene „transzendentale“ Denkfigur, die Heidegger ablehnt, angewiesen bleibt. Das „Sein selbst“ kann keine Kultur oder ihren Zustand „begründen“.

Heideggers Denken ist ergebnis- und gegenstandslos. Das bedeutet nicht, dass es keine Themen bespricht. Die späten Themen des „Ge-Stells“ und des „Ge-Vierts“ beanspruchen dieses Denken über Jahrzehnte hinweg. Doch die „innerste Be-wēgnis des Denkens“ verhindert und verweigert eine Dogmatisierung etwaiger Resultate dieser Thematisierungen. Wer bei Heidegger Lehren finden will, ignoriert die Ziel- und Gegenstandlosigkeit dieses Denkens. Die einzige Lehre, die es möglicherweise gibt, wäre die, dass es keine gibt und geben kann.

Ein Einwand liegt nahe. Der Gegenstand des vorliegenden Textes sind Heideggers Überlegungen $\mathrm{zu}$ einem gegenstands- und ziellosen Denken. Schließlich hat Heidegger sie niedergeschrieben, demnach eine gegenständliche Spur hinterlassen. Hätte Heidegger „nur“ gedacht und wie Sokrates die Schrift vermieden, darüber hinaus auch keinen Platon gehabt, würde das Denken erst wahrhaft gegenstands- und ziellos sein. So lange es sich äußert, bleibt es gegenständlich.

Heidegger hat sich mit der Schrift und ihrer Bedeutung für sein Denken vor allem in den Schwarzen Heften beschäftigt und die „Schrift der dichtenden Sage des ,Seyns““ als das „Handwerk des Denkens“ bezeichnet (Heidegger, 2015, p. 118). Diese Schrift ist für Heidegger die „Handschrift“ (p. 347); ein Gedanke, der ohne Zweifel mit dem Sachverhalt zusammenhängt, dass Heidegger alles, was er verfasste, mit der Hand schrieb. Eine Schreibmaschine benutzte er nie.

Ich will mich auf die Diskussion über die Eigenschaften und etwaigen Vorzüge der Handschrift -jenseits der Graphologie selbstverständlich-, oder über die technischen Eigenschaften der Maschinenschrift nicht einlassen. Wichtiger ist, dass Heidegger hinzufügt: „wenn sie der Nachschrift des Seyns in die Sprache hilft“ (p. 347). Indem die Handschrift „Nachschrift des Seyns“ ist, entzieht Heidegger ihr die Eigenständigkeit. Der schreibende Philosoph „entspricht“ einer anderen Schrift: „Jede echte Schrift ist Nachschrift. Sie schreibt nicht eine andere ab, doch sagt sie, indem sie der Sache entspricht" (p. 346). Und dann heißt es weiter: „Vielleicht ist die wesenhafte Schrift sehr selten; nicht weil der Mensch sie selten leistet, sondern weil die Nach-schrift als Sage des Seyns im Seyn selber gespart bleibt". 
Die „Sache“, der die Schrift des Denkens „entspricht“, ist das „Seyn selber“. In ihm bleibe die „Nach-schrift als Sage des Seyns gespart“. Die „Nach-schrift“ ist so nicht nur keine Abschrift. Sie ist zudem auch niemals erschöpfend in dem Sinne, dass das „Seyn selber“ zur Schrift werden könnte. Das „Seyn selber“ ist unerschöpflich in seinem Sinn, in seinen Bedeutungen. Dass Heidegger es in so vielen graphischen Formen darstellen konnte, ist ein Hinweis darauf.

Daraus entsprang für Heidegger ein ganzes Schrift-Feld, auf dem (sich) sein Denken in allen Richtungen ,be-wëgen“ kann. In allen Bewegungen bedeutet, dass Heidegger sein Denken nie nur in eine Richtung schriftlich entfaltet hat. Er arbeitete zuweilen an auch verschiedenen Manuskripten gleichzeitig; dieses Arbeiten, das in der Tat mehr mit der Gestaltung eines Feldes als mit der Gestaltung eines „Werkes“ zu tun hatte.

Die Gegenstands- und Ziellosigkeit des Denkens antwortet auf die Unerschöpflichkeit der Bedeutungen im „Seyn selber“. Es „,be-wëgt“ sich in ihm, indem es seine Unerschöpflichkeit „nach-schreibt“. Dieses „Be-wëgen“ ist so offen, dass Heidegger sogar dem „Grammatischen“" seinen regulierenden Einfluss auf die Schrift streitig macht (p. 358) ${ }^{10}$. Heideggers Forderung der Offenheit des Denkens und Schreibens lässt sich selbst an der Grammatik, dieser für ihn römischen Regulierung der Sprache, nicht begrenzen. Die oft belächelten Eigentümlichkeiten der Heideggerschen Sprache entspringen einer bewussten Entscheidung gegen dieses Regelwerk.

Wenn das „Seyn selber“ alle möglichen Schriften in sich „spart“, ist jede gegenständliche Schrift nichts weiter als ,,vor-läufig“. Sie als eine endgültige Bedeutung des Gesparten zu verstehen, wäre ein gewaltsamer Fehler, eine nicht zu rechtfertigende Behauptung. Und weil das gegenstands- und ziellose Denken über die Schrift sich ihrer Unerschöpflichkeit überlässt, neigt es zur Hyperbolé. So heißt es einmal in Heideggers Idiom:

Schreibt die Sage des Denkens ihr Gedachtes für die Leser? Oder ist ihre Nachschrift anfänglich die Inschrift des Seyns?, als welche es sich in die eigentliche Vergessenheit des Brauchs einschreibt? Die Inschrift weste dann selber als die Enteignis im Unter-schied (p. 401).

$\overline{10}$ „Dem Grammatischen bleibt das Wesen der Schrift als der Nach-schrift des Seyns gerade verborgen“. 
Die philosophische Schrift adressiert sich nicht an „Leser“, sondern vielmehr ans „Seyn selber“, dessen ,anfängliche Nachschrift“ die „Inschrift des Seyns" sei. Die „Inschrift“" aber schreibt sich nicht in irgendeinen öffentlichen Diskurs ein, sondern ,in die eigentliche Vergessenheit des Brauchs“, d.h. in die Verborgenheit des „Ereignisses“, in die „Enteignis im Unter-schied“. Diese extreme Überspitzung des gegenstands- und ziellosen Denkens als „Inschrift des Seyns“ jenseits aller „Leser“ ist eine jener Gesten der Heideggerschen Philosophie, die scheinbar unbemerkt von ihm in einen Dogmatismus umschlagen; in den Dogmatismus des einzigen lebenden Denkers, der von der „Inschrift des Seyns“" weiß -Heidegger, der Pseudo-Prophet. Und doch muss gerade für die hyperbolischen Ausbrüche dieses Denkens betont werden, dass sie dem Charakter des „Be-wëgens" nicht widersprechen. Auch die Hyperbolé ist ein Weg. Heidegger wählt ihn bewusst und verlässt ihn wieder.

Aus der „Frage nach dem Sinn von Sein“ ist das „Ereignis“ und das „Seyn selbst" und das "Seyn" und noch anderes entstanden. Sie entfalten den Raum und die Zeit, in dem und der (sich) das gegenstands- und ziellose Denken „be-wëgen“ kann, in der es rhetorisch und antirhetorisch, destruktiv und dogmatisch, poetisch und antipoetisch, exoterisch und esoterisch, wissenschaftlich und antiwissenschaftlich, metapolitisch und apolitisch, christlich und antichristlich, ethisch und antiethisch sowie philosophisch und antiphilosophisch erscheinen kann. Diese Offenheit des Denkens versucht Heideggers Philosophieren zu praktizieren und praktizierend deutlicher werden zu lassen.

Zurück zum Anfang. Die Geschichte der Philosophie ist eine der Vergegenständlichungen von Bedeutung. Sie manifestiert sich in Texten, die zu „Werken“ werden, „Diskurse“ mit „Argumenten“ beliefern. Daraus ergibt sich eine institutionelle Form der Philosophie und des Philosophierens, die sich auch in ihren Popularisierungen fortsetzt. Wenn im akademischen Studium das „Werk“ als Studien- und Forschungsgegenstand dient, so in der Popularisierung der Philosophie als Ware. Das alles gibt der Philosophie eine materielle Plattform, auf die sich das Denken beziehen kann, indem es selbst an ihr baut ${ }^{11}$.

${ }^{11}$ Heideggers Idiosynkrasie im Verhältnis zur Öffentlichkeit ist bekannt. So schreibt er einmal: „Das Kesseltreiben gegen mein Denken, d.h. gegen die Vorstellung, die man sich davon gemacht hat, verstärkt sich überall her und nimmt die Form der Organisation an. Die Einkesselung muß bei solchem 
Diese Tendenz der Philosophie zum Gegenstand schlägt sich nicht nur in der Reflexion, Lehre und Rekonstruktion ihrer Text-Geschichten nieder, sondern darüber hinaus in der Anknüpfung des Philosophierens an bestehende gesellschaftliche und politische „Diskurse“ wie dem Vormarsch der KI- und Gehirn-Forschung, dem Rassismus, der Geschlechtertheorie oder dem Corona-Virus etc. Hier nun wird die Philosophie zu einem sich verobjektivierenden Kommentar von Objekten, der wiederum in Texten seine eigene Geschichte verobjektiviert. Das massivste Objekt einer solchen Philosophie scheint übrigens die Gesellschaft zu sein, in der „Debatten“" entstehen und vergehen.

Es wäre zu einfach, diese Materialisierung der Philosophie als Verfallsphänomen oder als narzisstische Schwäche zu interpretieren. Das Begehren nach Präsenz und Permanenz hat tiefere Ursachen als die Ökonomie des Narzissmus, die allerdings ihr Resonanzraum ist. Heidegger hat das in seinen Auslegungen der von ihm als „Metaphysik“ bezeichneten Epoche des Philosophierens herauszubekommen versucht. Aber trotz seiner Hinweise auf das „Ge-Stell“" bleibt die Frage nach dem Vorrang einer bestimmten Auffassung der Wirklichkeit (Realität) und ihrer Bedingungen bestehen. Warum integriert sich die Philosophie so mehr oder weniger unreflektiert in die bestehenden Strukturen?

Dass all dem ein nichtgegenständliches Ereignis -das Denken- vorausgeht, weiß jeder und jede - und doch wird ihm als solchem philosophisch kaum Aufmerksamkeit geschenkt. Gewiss, Platon oder Hegel haben ,gedacht", bevor und indem sie ihre Texte produzierten, aber das scheint wie eine Banalität keine Rolle zu spielen. Doch ist es wirklich nur eine Banalität? An diesem Punkt setzt Heideggers Verständnis des Denkens an. Er versucht, genau dieses vor aller Verobjektivierung liegende Geschehnis zu betonen, indem er es -so weit als möglich- von jener abzulösen versucht.

Damit gerät eine bestimmte, sehr mächtige Form der Philosophie ins Wanken. Jede Sedimentierung des Denkens in „Werken“ und „Diskursen“ erweist sich als eine Verstellung und als ein Abbau von Offenheit. Ein Philosophieren, das sich in Resultaten von Erkenntnisprozessen darstellt, hat sich den

Umtrieb gelingen. Aber man wird den Kessel -leer finden“ (Heidegger, 2020a, p. 29). Ob es eine Jagd auf Heideggers Denken gegeben hat, mag dahingestellt bleiben. Doch der Hinweis auf die Unmöglichkeit, sein Denken gleichsam dingfest zu machen, ist bemerkenswert. 
Notwendigkeiten der Institutionalisierung und der Ökonomie unterworfen. Wo man Studenten und Studentinnen Denken lehren sollte, lehrt man sie scheinbar feststehende Kenntnisse aus der Geschichte der Philosophie. Selbst wo zurecht eingeräumt wird, dass Beides zusammengehört, wird das Denken selbst nicht weiter thematisiert ${ }^{12}$.

Andererseits dürfte die Radikalität, mit der Heidegger jede Vergegenständlichung des Denkens, ja auch nur jede Verknüpfung mit bestehenden Institutionen und Öffentlichkeiten (jedenfalls im Denken der Schwarzen Hefte und der zu seiner Zeit unveröffentlichten „seinsgeschichtlichen Abhandlungen") das bleiben, als was sie sich selbst auch häufig beschrieben hat, nämlich als ,einzigartig“"13. Die Entscheidung, das Denken in dieser Weise dem gegenstands- und ziellosen „Be-wëgen“ $\mathrm{zu}$ überlassen, ist $\mathrm{zu}$ individuell, als dass sie unmittelbar übernommen werden könnte. Zudem würde eine solche Mimesis Heideggers eigentliche Motivation der Entscheidung konterkarieren: Ihr antidogmatischer Charakter würde zum Dogma.

Und dennoch legt Heideggers Denken den Finger in die Wunde. Die Auffassung, in der Philosophie ginge es vorzüglich um zu lehrende und $\mathrm{zu}$ studierende „Werke“, arbeitet der Institutionalisierung und Ökonomisierung bzw. Popularisierung der Philosophie vor. Diese wiederum fördern jene Auffassung, so dass das Innerste der Philosophie, das „Denken selbst“, immer mehr in Vergessenheit gerät. Das wird sich schließlich auf ihre Vergegenständlichungen im „Werk“ selbst auswirken. Inzwischen scheinen die „Werke“ selber nur noch eine Reminiszenz dessen zu sein, was sie einmal waren. Die Philosophie ist ein „Schatten in einer Höhle“ (Nietzsche, 1980, p. 467) geworden.

\footnotetext{
${ }^{12}$ Man könnte das hier dargestellte Problem auch im Kontext der von Heidegger immer wieder thematisierten „Logik“ aufzäumen. Heidegger arbeitete früh an einer Destruktion der traditionellen Logik, um mit der Dichtung eine andere zu formulieren (Heidegger, 2020b, p. 10). Die späteren Erläuterungen zum „Denken“ bilden -objektiv betrachtet- mit diesen früheren Gedanken zur „Logik“ einen Zusammenhang.

13 „Denken ist in dem einzigartigen Sinne; denn es ist das, was dem Wesen des Seyns, dem Ereignis des Brauchs ent-springt und innerhalb seiner in den Brauch, ihn hütend, und für ihn ein-springt" (Heidegger, 2015, p. 398).
} 


\section{Verweise}

Chighel, M. (2020). Kabale. Das Geheimnis des hebräischen Humanismus im Lichte von Heideggers Denken. Frankfurt a. M.: Vittorio Klostermann.

Derrida, J. (1983). Grammatologie (Übers. Rheinberger, H. J. und Zischler, H.). Frankfurt a. M.: Suhrkamp.

Faye, E. (2005). Heidegger: l'introduction du nazisme dans la philosophie. Paris: Albin Michel.

Heidegger, M. (1976). Was ist Metaphysik? von Herrmann, F. W. (Hrsg.). Wegmarken(GA9). (pp. 103-122). Frankfurta.M.: Vittorio Klostermann.

Heidegger, M. (1977). Sein und Zeit (GA 2). von Herrmann, F. W. (Hrsg.). Frankfurt a. M.: Vittorio Klostermann.

Heidegger, M. (1978). Frühe Schriften (GA 1). von Herrmann, F. W. (Hrsg.). Frankfurt a. M.: Vittorio Klostermann.

Heidegger, M. (1985). Unterwegs zur Sprache (GA 12). von Herrmann, F. W. (Hrsg.). Frankfurt a. M.: Vittorio Klostermann.

Heidegger, M. (1993). Grundprobleme der Phänomenologie (1919/20) (GA 58). Gander, H. (Hrsg.). Frankfurt a. M.: Vittorio Klostermann.

Heidegger, M. (1999). Zur Bestimmung der Philosophie (GA 56/57). Heimbüchel, B. (Hrsg.). Frankfurt a. M.: Vittorio Klostermann.

Heidegger, M. (2007). Zur Sache des Denkens (GA 14). von Herrmann, F. W. (Hrsg.). Frankfurt a. M.: Vittorio Klostermann.

Heidegger, M. (2012). Der Anfang der abendländischen Philosophie. Auslegung des Anaximander und Parmenides (GA 32). Trawny, P. (Hrsg.). Frankfurt a. M.: Vittorio Klostermann.

Heidegger, M. (2015) Anmerkungen I-V (GA 97). Trawny, P. (Hrsg.). Frankfurt a. M.: Vittorio Klostermann.

Heidegger, M. (2017). Zu eigenen Veröffentlichungen (GA 82). von Herrmann, F. W. (Hrsg.). Frankfurt a. M.: Vittorio Klostermann.

Heidegger, M. (2019). Vigiliae und Notturno (Schwarze Hefte 1952/53 bis 1957) (GA 100). Trawny, P. (Hrsg.). Frankfurt a. M.: Vittorio Klostermann. 
Heidegger, M. (2020a). Winke I und II (Schwarze Hefte 1957 bis 1959) (GA 101). Trawny, P. (Hrsg.). Frankfurt a. M.: Vittorio Klostermann.

Heidegger, M. (2020b). Logik als die Frage nach dem Wesen der Sprache (GA 36). Trawny, P. (Hrsg.). Frankfurt a. M.: Vittorio Klostermann.

Marx, K \& Engels, F. (2010). Manifest der Kommunistischen Partei. Grundsätze des Kommunismus. Stuttgart: Reclam.

Nietzsche, F. (1980). Die fröhliche Wissenschaft (KSA 3). Kritische Studien Ausgabe. Colli G. und Montinari, M. (Hrsg.). Berlin: Walter de Gruyter.

Platon (2017). Phaidros. (Übers. Schleiermacher, F.). Altenmünster: Jazzybee Verlag.

Pöggeler, O. (1963). Der Denkweg Martin Heideggers. Pfullingen: Günther Neske.

Trawny, P. (2016). Martin Heidegger -Eine kritische Einführung. Frankfurt a. M.: Vittorio Klostermann.

Trawny, P. (2019). Heidegger-Fragmente. Eine philosophische Biographie. Frankfurt a. M.: Fischer Verlag.

Vietta, S. (2015). „Etwas rast um den Erdball...“. Martin Heidegger: Ambivalente Existenz und Globalisierungskritik. Paderborn: Fink Verlag.

von Herrmann, F. W. und Alfieri, F. (2017). Die Wahrheit über die „, Schwarzen Hefte“. Berlin: Duncker \& Humblot.

Zimmermann, H. D. (2016). Martin und Fritz Heidegger. Freiburg im Breisgau: Herder Verlag. 